\title{
Effects of Six Kinds of Sodium-Glucose Cotransporter 2 Inhibitors on Metabolic Parameters, and Summarized Effect and Its Correlations With Baseline Data
}

\author{
Hidekatsu Yanai $^{\mathrm{a}, \mathrm{b}}$, c, Mariko Hakoshima ${ }^{\mathrm{a}}$, Hiroki Adachi ${ }^{\mathrm{a}}$, Akiko Kawaguchi ${ }^{\mathrm{a}}$, Yoko Waragai ${ }^{\mathrm{a}}$, \\ Tadanao Harigae ${ }^{a}$, Yoshinori Masui ${ }^{a}$, Kouki Kakuta ${ }^{a}$, Hidetaka Hamasakia \\ Hisayuki Katsuyama ${ }^{\mathrm{a}}$, Tomoko Kaga ${ }^{\mathrm{b}}$, Akahito Sako ${ }^{\mathrm{a}, \mathrm{b}}$
}

\begin{abstract}
Background: Sodium-glucose cotransporter 2 inhibitor (SGLT2i) blocks reabsorption of glucose by inhibiting SGLT2 in kidney, promotes the renal excretion of glucose and improves blood glucose control without requiring insulin secretion. Anti-atherosclerotic effects of SGLT2is have not been fully elucidated until today.
\end{abstract}

Methods: We retrospectively picked up patients with type 2 diabetes who had been continuously prescribed SGLT2i for 3 months or more between April 2014 and December 2016 by a chart-based analysis, and compared metabolic parameters including coronary risk factors before the SGLT2i treatment with the data at 3 and 6 months after the SGLT2i treatment started.

Results: We found 26 patients treated with tofogliflozin, 34 patients with canagliflozin, 27 patients with empagliflozin, 23 patients with ipragliflozin, 68 patients with dapagliflozin and 71 patients with luseogliflozin. Each SGLT2i ameliorated metabolic parameters, in different patterns. SGLT2is reduced body weight, systolic and diastolic blood pressures, plasma glucose, hemoglobin A1c, aspartate aminotransferase, alanine aminotransferase, $\gamma$-glutamyltransferase, uric acid, triglyceride and non-high-density lipoprotein-cholesterol (HDL-C), and elevated HDL-C; however, they did not affect LDLcholesterol levels. Change in each metabolic parameter was significantly correlated with each metabolic parameter at baseline.

Conclusion: The present study demonstrated that SGLT2i ameliorated body weight, blood pressure, liver function, serum lipids and uric acid, in addition to improvement of glucose metabolism in patients

Manuscript accepted for publication April 20, 2017

aDepartment of Internal Medicine, National Center for Global Health and Medicine Kohnodai Hospital, Chiba, Japan

${ }^{\mathrm{b}}$ Clinical Research and Trial Center, National Center for Global Health and Medicine Kohnodai Hospital, Chiba, Japan

${ }^{\mathrm{c} C}$ Corresponding Author: Hidekatsu Yanai, Department of Internal Medicine, National Center for Global Health and Medicine Kohnodai Hospital, 1-7-1 Kohnodai, Ichikawa, Chiba 272-0034, Japan.

Email: dyanai@hospk.ncgm.go.jp

doi: https://doi.org/10.14740/jocmr3046w with type 2 diabetes.

Keywords: Body weight; Hemoglobin A1c; Serum lipids; Sodiumglucose cotransporter 2 inhibitor; Uric acid

\section{Introduction}

Sodium-glucose cotransporter 2 (SGLT2) is expressed in the proximal tubule of kidney and mediates reabsorption of glucose [1], and SGLT2 inhibitor (SGLT2i) blocks reabsorption of glucose by inhibiting SGLT2, promotes the renal excretion of glucose and improves blood glucose control without requiring insulin secretion [2].

We previously proposed the possible anti-atherosclerotic effects beyond glucose lowering of SGLT2i [3]. Briefly, caloric loss by SGLT2 inhibition may decrease plasma glucose without increasing insulin secretion, which may reduce body weight and result in improvement of insulin resistance. Improvement of insulin resistance may ameliorate atherosclerotic risk factors such as dyslipidemia, hypertension and elevated inflammatory cytokines [3]. Osmotic diuretics by SGLT2 inhibition may also decrease blood pressure (BP), favorably affecting atherosclerosis [3].

Actually, EMPA-REG OUTCOME, a randomized placebo-controlled trial (RCT) that examined the effect of empagliflozin in addition to standard of care in patients with type 2 diabetes and established cardiovascular (CV) disease demonstrated a significant reduction in the incidence of $\mathrm{CV}$ death and heart failure hospitalization [4].

However, whether SGLT2i is associated with reduction in myocardial infarction or not, or whether the beneficial effect observed with empagliflozin in EMPAREG OUTCOME study is a class effect or not, remained to be controversial [5-8].

Anti-atherosclerotic effects of SGLT2i have not been fully elucidated until today. Our institute, National Center for Global Health and Medicine (NCGM), is the National Center which has the discovery of the best therapy for diabetes as our mission, and has many specialists for diabetes treatment. To elucidate anti-atherosclerotic effects of SGLT2i, we retrospectively studied six kinds of SGLT2is (tofogliflozin, canagliflo- 
Table 1. Changes in Metabolic Parameters at 3 and 6 Months After the Start of Tofogliflozin

\begin{tabular}{|c|c|c|c|c|c|c|}
\hline & $\mathbf{n}$ & $\begin{array}{l}\text { Baseline, } \\
\text { mean } \pm \text { SD }\end{array}$ & $\begin{array}{l}3 \text { months after, } \\
\text { mean } \pm \text { SD }\end{array}$ & $\mathbf{n}$ & $\begin{array}{l}\text { Baseline, } \\
\text { mean } \pm \mathrm{SD}\end{array}$ & $\begin{array}{l}6 \text { months after, } \\
\text { mean } \pm \text { SD }\end{array}$ \\
\hline Body weight (kg) & 12 & $85.9 \pm 15.1$ & $83.1 \pm 13.2^{* *}$ & 11 & $81.5 \pm 12.4$ & $79.1 \pm 11.3 * *$ \\
\hline Systolic BP (mm Hg) & 7 & $124.0 \pm 12.9$ & $123.9 \pm 7.5$ & 8 & $127.3 \pm 15.1$ & $126.4 \pm 11.0$ \\
\hline Diastolic BP (mm Hg) & 7 & $73.6 \pm 6.1$ & $74.3 \pm 6.8$ & 8 & $76.0 \pm 8.9$ & $76.9 \pm 5.2$ \\
\hline Plasma glucose (mg/dL) & 19 & $214.7 \pm 91.0$ & $189.1 \pm 76.2$ & 16 & $217.6 \pm 95.7$ & $191.6 \pm 67.5$ \\
\hline HbAlc (\%) & 13 & $8.6 \pm 1.5$ & $7.8 \pm 1.5^{* *}$ & 12 & $8.7 \pm 1.4$ & $7.9 \pm 1.2 *$ \\
\hline AST (U/L) & 19 & $28.1 \pm 14.2$ & $23.7 \pm 7.8^{*}$ & 16 & $30.9 \pm 18.0$ & $28.7 \pm 18.7$ \\
\hline $\operatorname{ALT}(\mathrm{U} / \mathrm{L})$ & 19 & $33.8 \pm 27.3$ & $26.6 \pm 11.8$ & 16 & $36.8 \pm 29.4$ & $34.9 \pm 33.7$ \\
\hline$\gamma-\mathrm{GT}(\mathrm{U} / \mathrm{L})$ & 11 & $36.4 \pm 22.4$ & $29.9 \pm 14.3$ & 10 & $46.1 \pm 30.1$ & $40.1 \pm 26.2 *$ \\
\hline Uric acid (mg/dL) & 19 & $5.3 \pm 1.2$ & $5.0 \pm 1.1$ & 16 & $5.3 \pm 1.2$ & $4.9 \pm 0.9 *$ \\
\hline $\mathrm{TG}(\mathrm{mg} / \mathrm{dL})$ & 17 & $216.6 \pm 114.4$ & $214.1 \pm 188.8$ & 16 & $219.4 \pm 118.5$ & $204.1 \pm 123.8$ \\
\hline HDL-C (mg/dL) & 17 & $50.4 \pm 10.5$ & $51.8 \pm 9.4$ & 16 & $50.1 \pm 10.0$ & $52.8 \pm 8.9$ \\
\hline LDL-C (mg/dL) & 16 & $113.8 \pm 39.4$ & $112.7 \pm 36.1$ & 15 & $112.4 \pm 39.6$ & $108.2 \pm 28.4$ \\
\hline Non-HDL-C (mg/dL) & 7 & $139.9 \pm 24.2$ & $137.9 \pm 22.3$ & 5 & $135.2 \pm 22.9$ & $142.4 \pm 29.2$ \\
\hline
\end{tabular}

${ }^{*} P<0.1$ and ${ }^{* *} P<0.05$ vs. baseline.

zin, empagliflozin, ipragliflozin, dapagliflozin and luseogliflozin) and also summarized effects of all SGLT2is on metabolic parameters including coronary risk factors in patients with type 2 diabetes. Further, we studied the most crucial factor at baseline to determine the changes in metabolic parameters due to SGLT2is.

\section{Materials and Methods}

This study was approval by the Institutional Ethics Committee in National Center for Global Health and Medicine, and was also performed in accordance with the Declaration of Helsinki. We selected patients with type 2 diabetes who had been prescribed the standard-dose of SGLT2 is for 3 months or longer between April 2014 and December 2016 based on medical charts. We compared the data at baseline and at 3 and 6 months after the start of SGLT2i. Body weight, BP, plasma glucose, hemoglobin A1c (HbA1c), serum low-density lipoprotein-cholesterol (LDL-C), triglyceride (TG), high-density lipoprotein-cholesterol (HDL-C), uric acid, aspartate aminotransferase (AST), alanine aminotransferase (ALT) and $\gamma$-glutamyltransferase $(\gamma-\mathrm{GT})$ in studied subjects were measured almost at the same time point at the baseline and 3 or 6

Table 2. Changes in Metabolic Parameters at 3 and 6 Months After the Start of Canagliflozin

\begin{tabular}{lllllll}
\hline & n & $\begin{array}{l}\text { Baseline, } \\
\text { mean } \pm \text { SD }\end{array}$ & $\begin{array}{l}\text { 3 months after, } \\
\text { mean } \pm \text { SD }\end{array}$ & n & $\begin{array}{l}\text { Baseline, } \\
\text { mean } \pm \text { SD }\end{array}$ & $\begin{array}{l}\text { 6 months after, } \\
\text { mean } \pm \text { SD }\end{array}$ \\
\hline Body weight (kg) & 21 & $79.4 \pm 12.9$ & $78.5 \pm 13.3^{*}$ & 19 & $80.3 \pm 13.1$ & $78.8 \pm 13.3^{*}$ \\
\hline Systolic BP (mm Hg) & 23 & $131.6 \pm 12.1$ & $126.0 \pm 17.3^{*}$ & 21 & $131.9 \pm 28.7$ & $120.6 \pm 15.4 * *$ \\
\hline Diastolic BP (mm Hg) & 23 & $76.9 \pm 10.1$ & $76.4 \pm 10.7$ & 21 & $77.4 \pm 10.8$ & $72.0 \pm 10.4 * *$ \\
\hline Plasma glucose (mg/dL) & 27 & $214.3 \pm 75.9$ & $175.4 \pm 72.7 * *$ & 27 & $210.9 \pm 74.7$ & $170.0 \pm 49.7 * *$ \\
HbA1c (\%) & 25 & $8.6 \pm 1.6$ & $7.7 \pm 1.1 * *$ & 24 & $8.7 \pm 1.6$ & $7.7 \pm 1.0^{* *}$ \\
AST (U/L) & 27 & $30.9 \pm 33.2$ & $23.9 \pm 11.1$ & 27 & $30.1 \pm 33.2$ & $23.7 \pm 10.5$ \\
ALT (U/L) & 26 & $41.7 \pm 49.1$ & $34.6 \pm 28.2$ & 26 & $40.5 \pm 49.2$ & $30.3 \pm 20.2$ \\
$\gamma$-GT (U/L) & 22 & $37.5 \pm 24.0$ & $30.8 \pm 18.9 * *$ & 23 & $36.7 \pm 23.7$ & $31.3 \pm 21.8^{*}$ \\
\hline Uric acid (mg/dL) & 25 & $5.3 \pm 1.4$ & $5.1 \pm 1.2$ & 26 & $5.0 \pm 1.1$ & $5.0 \pm 1.1$ \\
TG (mg/dL) & 25 & $196.0 \pm 135.7$ & $166.9 \pm 104.2 *$ & 26 & $193.5 \pm 133.5$ & $175.3 \pm 125.2$ \\
\hline HDL-C (mg/dL) & 25 & $50.9 \pm 12.8$ & $50.3 \pm 11.9$ & 26 & $51.8 \pm 13.4$ & $51.3 \pm 14.0$ \\
\hline LDL-C (mg/dlL) & 24 & $102.6 \pm 29.1$ & $104.3 \pm 26.3$ & 25 & $103.1 \pm 28.6$ & $109.2 \pm 29.2$ \\
\hline Non-HDL-C (mg/dL) & 19 & $134.3 \pm 36.4$ & $129.2 \pm 29.6$ & 19 & $135.3 \pm 36.4$ & $135.9 \pm 33.9$ \\
\hline
\end{tabular}

${ }^{*} \mathrm{P}<0.1$ and ${ }^{* *} \mathrm{P}<0.05$ vs. baseline. 
Table 3. Changes in Metabolic Parameters at 3 and 6 Months After the Start of Empagliflozin

\begin{tabular}{|c|c|c|c|c|c|c|}
\hline & $\mathbf{n}$ & $\begin{array}{l}\text { Baseline, } \\
\text { mean } \pm \text { SD }\end{array}$ & $\begin{array}{l}3 \text { months after, } \\
\text { mean } \pm \text { SD }\end{array}$ & $\mathbf{n}$ & $\begin{array}{l}\text { Baseline, } \\
\text { mean } \pm \mathrm{SD}\end{array}$ & $\begin{array}{l}6 \text { months after, } \\
\text { mean } \pm \text { SD }\end{array}$ \\
\hline Body weight (kg) & 17 & $87.5 \pm 21.4$ & $86.6 \pm 21.3$ & 6 & $84.9 \pm 30.9$ & $80.2 \pm 27.9^{* *}$ \\
\hline Systolic BP (mm Hg) & 16 & $135.4 \pm 17.9$ & $128.1 \pm 14.8$ & 4 & $147.0 \pm 31.4$ & $146.3 \pm 35.8$ \\
\hline Diastolic BP (mm Hg) & 16 & $81.0 \pm 16.0$ & $78.6 \pm 11.8$ & 4 & $84.8 \pm 23.9$ & $88.0 \pm 28.2$ \\
\hline Plasma glucose (mg/dL) & 17 & $222.4 \pm 73.0$ & $171.5 \pm 66.0^{*}$ & 6 & $205.7 \pm 77.5$ & $145.5 \pm 38.6^{* *}$ \\
\hline HbA1c (\%) & 17 & $9.4 \pm 2.0$ & $7.6 \pm 1.1 * *$ & 6 & $9.6 \pm 2.6$ & $7.4 \pm 1.0^{* *}$ \\
\hline $\operatorname{AST}(\mathrm{U} / \mathrm{L})$ & 17 & $31.2 \pm 21.3$ & $28.1 \pm 17.8$ & 6 & $24.8 \pm 18.6$ & $20.3 \pm 7.1$ \\
\hline ALT (U/L) & 17 & $46.2 \pm 43.5$ & $39.6 \pm 34.6$ & 6 & $27.0 \pm 21.1$ & $20.0 \pm 9.4$ \\
\hline$\gamma$-GT (U/L) & 16 & $104.6 \pm 213.6$ & $73.9 \pm 127.7$ & 5 & $29.0 \pm 20.4$ & $23.0 \pm 9.8$ \\
\hline Uric acid (mg/dL) & 15 & $5.5 \pm 2.2$ & $5.1 \pm 1.6$ & 6 & $4.4 \pm 1.7$ & $5.2 \pm 3.1$ \\
\hline TG (mg/dL) & 17 & $257.6 \pm 204.3$ & $173.3 \pm 105.4^{* *}$ & 6 & $164.0 \pm 98.8$ & $104.7 \pm 36.7$ \\
\hline HDL-C (mg/dL) & 17 & $51.1 \pm 16.5$ & $52.5 \pm 16.5$ & 6 & $57.3 \pm 24$ & $63.0 \pm 23.5^{* *}$ \\
\hline LDL-C (mg/dL) & 17 & $111.0 \pm 29.1$ & $102.6 \pm 24.8$ & 6 & $113.7 \pm 36.6$ & $107.2 \pm 28.2$ \\
\hline Non-HDL-C (mg/dL) & 15 & $159.4 \pm 50.6$ & $134.9 \pm 30.3^{* *}$ & 5 & $145.4 \pm 47.8$ & $119.0 \pm 28.9$ \\
\hline
\end{tabular}

${ }^{*} \mathrm{P}<0.1$ and ${ }^{* *} \mathrm{P}<0.05$ vs. baseline.

months after the start of SGLT2 inhibitors. LDL-C levels were determined by the direct measurement or by the Friedewald's formula. Estimated glomerular filtration rate (eGFR) was calculated by a modified three variable equation for estimating GFR in Japanese patients [9].

Comparison of the variables determined before and after was analyzed by a paired Student's $t$-test. Spearman's correlation was performed to determine the correlations between the data before the start of SGLT2 $i$ treatment and changes in variables after the SGLT2i treatment. All data are expressed as mean $\pm \mathrm{SD} . \mathrm{P}<0.05$ and $\mathrm{P}<0.1$ were considered to be statistically significant and to show tendency, respectively.

\section{Results}

We found 26 patients treated with tofogliflozin (age, $54.0 \pm$ 11.5 years; male/female, 11/15), 34 patients with canagliflozin (56.6 \pm 16.2 years; male/female, 16/18), 27 patients with empagliflozin $(51.0 \pm 9.2$ years; male/female, $15 / 12), 23$ patients with ipragliflozin $(50.0 \pm 14.0$ years; male/female, 9/14), 68 patients with dapagliflozin $(52.3 \pm 13.4$ years; male/female, $35 / 33$ ) and 71 patients with luseogliflozin (54.4 \pm 13.4 years; male/female, 42/29). Total 249 patients had been treated by SGLT2 $\mathrm{i}$ at least for 3 months.

Changes in metabolic parameters at 3 and 6 months after the start of each SGLT2i were shown in Tables 1-6. Tofogliflozin significantly reduced body weight at both 3 and 6 months after the start, and significantly reduced $\mathrm{HbAlc}$ at 3 months and tended to reduce HbAlc at 6 month (Table 1). AST at 3 months, $\gamma$-GT and uric acid at 6 months tended to decrease from baseline. Canagliflozin tended to reduce body weight at 3 and 6 months, and systolic BP at 3 months (Table 2). Both systolic and diastolic BP significantly decreased by canagliflozin at 6 month. Canagliflozin significantly reduced plasma glucose and HbA1c at both 3 and 6 months. Serum $\gamma-\mathrm{GT}$ and TG tended to decrease at 3 months, and $\gamma$-GT significantly decreased, from baseline, at 6 months. Empagliflozin significantly reduced body weight, plasma glucose and $\mathrm{HbA1c}$, and significantly increased HDL-C at 6 months (Table 3). HbA1c, TG and non-HDL-C significantly decreased from baseline at 3 months. Ipragliflozin significantly reduced body weight and HbAlc at 3 months, and tended to reduce body weight at 6 months (Table 4). HbA1c and AST significantly decreased from baseline at 6 months. Dapagliflozin significantly reduced body weight and $\mathrm{HbA} 1 \mathrm{c}$ at 3 months, and reduced body weight, HbA1c, AST and ALT, and also increased HDL-C at 6 months (Table 5). Luseogliflozin significantly reduced body weight, diastolic BP, HbA1c, AST, ALT, $\gamma$-GT and uric acid at both 3 and 6 months. Systolic BP, plasma glucose, and TG tended to decrease from baseline at 3 months, and TG and non-HDL-C significantly decreased from baseline at 6 months (Table 6).

Changes in metabolic parameters at 3 and 6 months after the start of SGLT2 is were shown in Table 7, and the summary of changes due to each SGLT2i was shown in Table 8. SGLT2 is significantly reduced body weight, systolic BP, plasma glucose, HbA1c, AST, ALT and $\gamma$-GT at both 3 and 6 months, and significantly decreased diastolic BP, uric acid and nonHDL-C at 3 months. Serum TG at both 3 and 6 months, diastolic BP and non-HDL-C at 6 months tended to decrease, and HDL-C at 6 months tended to increase by SGLT2i treatment.

The most crucial determinant in parameters at baseline for changes in metabolic parameters due to SGLT2i treatment was shown in Table 9. Changes in systolic BP, diastolic BP, plasma glucose, HbA1c, AST, ALT, uric acid and LDL-C at both 3 and 6 months after the start of SGLT $2 i$ were most closely correlated with systolic BP, diastolic BP, plasma glucose, HbAlc, AST, ALT, uric acid and LDL-C at baseline, respectively. Changes in $\gamma$-GT at 3 months were most closely correlated 
Table 4. Changes in Metabolic Parameters at 3 and 6 Months After the Start of Ipragliflozin

\begin{tabular}{lllllll}
\hline & n & $\begin{array}{l}\text { Baseline, } \\
\text { mean } \pm \text { SD }\end{array}$ & $\begin{array}{l}\text { 3 months after, } \\
\text { mean } \pm \text { SD }\end{array}$ & n & $\begin{array}{l}\text { Baseline, } \\
\text { mean } \pm \text { SD }\end{array}$ & $\begin{array}{l}\text { 6 months after, } \\
\text { mean } \pm \text { SD }\end{array}$ \\
\hline Body weight $(\mathrm{kg})$ & 21 & $90.9 \pm 18.6$ & $89.5 \pm 18.1 * *$ & 13 & $95.2 \pm 20.9$ & $93.8 \pm 20.8 *$ \\
Systolic BP (mm Hg) & 17 & $130.5 \pm 14.7$ & $126.7 \pm 19.0$ & 15 & $127.3 \pm 8.9$ & $126.4 \pm 21.1$ \\
\hline Diastolic BP (mm Hg) & 17 & $77.5 \pm 10.3$ & $79.6 \pm 12.1$ & 15 & $74.3 \pm 9.2$ & $76.7 \pm 10.2$ \\
\hline Plasma glucose (mg/dL) & 23 & $176.1 \pm 77.4$ & $161.3 \pm 78.9$ & 19 & $176.8 \pm 80.4$ & $158.6 \pm 60.2$ \\
HbA1c (\%) & 19 & $8.2 \pm 1.6$ & $7.5 \pm 1.3 * *$ & 16 & $8.2 \pm 1.8$ & $7.4 \pm 1.1^{* *}$ \\
AST (U/L) & 20 & $31.4 \pm 19.7$ & $26.8 \pm 16.4$ & 16 & $33.5 \pm 21.1$ & $27.0 \pm 13.2 * *$ \\
ALT (U/L) & 22 & $44.0 \pm 39.0$ & $36.7 \pm 27.6$ & 18 & $47.6 \pm 42.0$ & $37.4 \pm 20.4$ \\
$\gamma$-GT (U/L) & 17 & $64.5 \pm 50.2$ & $61.6 \pm 46.7$ & 14 & $59.2 \pm 38.3$ & $58.9 \pm 38.2$ \\
Uric acid (mg/dL) & 16 & $5.5 \pm 1.4$ & $5.4 \pm 1.1$ & 14 & $5.4 \pm 1.4$ & $5.0 \pm 1.1$ \\
TG (mg/dL) & 22 & $176.0 \pm 69.6$ & $161.0 \pm 74.6$ & 18 & $177.0 \pm 76.2$ & $172.1 \pm 87.2$ \\
HDL-C (mg/dL) & 22 & $47.2 \pm 10.6$ & $46.7 \pm 13.0$ & 18 & $48.3 \pm 10.8$ & $47.2 \pm 13.0$ \\
\hline LDL-C (mg/dL) & 21 & $108.2 \pm 30.3$ & $105.6 \pm 34.4$ & 15 & $110.4 \pm 30.6$ & $102.5 \pm 30.8$ \\
Non-HDL-C (mg/dL) & 12 & $143.8 \pm 29.1$ & $140.3 \pm 30.4$ & 11 & $144.2 \pm 30.1$ & $134.5 \pm 30.4$ \\
\hline
\end{tabular}

${ }^{*} P<0.1$ and ${ }^{* *} P<0.05$ vs. baseline.

with $\gamma$-GT at baseline; however, the most crucial determinant of changes in $\gamma$-GT at 6 month was ALT. Changes in TG at 3 and 6 months were most closely correlated with non-HDL-C and TG at baseline, respectively. Changes in HDL-C at 3 and 6 months were most closely correlated with LDL-C and HDL-C at baseline, respectively, and changes in non-HDL-C at 3 and 6 months were most closely correlated with non-HDL-C and LDL-C at baseline, respectively. Changes in body weight at both 3 and 6 months were most closely correlated with systolic $\mathrm{BP}$ but not body weight at baseline. The change in body weight at 3 months was significantly correlated with body weight at baseline $(\mathrm{r}=-0.2 ; \mathrm{P}=0.022)$, however, that at 6 months was not significantly correlated with body weight at baseline $(\mathrm{r}=$ $-0.157 ; \mathrm{P}=0.125)$.

\section{Discussion}

SGLT2 $\mathrm{i}$ has been proved to be significantly associated with weight loss and reduction of BP, in addition to lowering plasma glucose, by a relatively large number of studies [3]. However, effects of SGLT2 $\mathrm{i}$ on other metabolic parameters including coronary risk factors such as serum lipids, uric acid and liver function remained to be unclear.

Table 5. Changes in Metabolic Parameters at 3 and 6 Months After the Start of Dapagliflozin

\begin{tabular}{|c|c|c|c|c|c|c|}
\hline & $\mathbf{n}$ & $\begin{array}{l}\text { Baseline, } \\
\text { mean } \pm \text { SD }\end{array}$ & $\begin{array}{l}3 \text { months after, } \\
\text { mean } \pm S D\end{array}$ & $\mathbf{n}$ & $\begin{array}{l}\text { Baseline, } \\
\text { mean } \pm \mathrm{SD}\end{array}$ & $\begin{array}{l}6 \text { months after, } \\
\text { mean } \pm \text { SD }\end{array}$ \\
\hline Body weight (kg) & 33 & $81.1 \pm 17.4$ & $79.5 \pm 17.5^{* *}$ & 27 & $78.5 \pm 19.6$ & $76.9 \pm 19.1 * *$ \\
\hline Systolic BP (mm Hg) & 30 & $130.2 \pm 14.5$ & $131.2 \pm 17.0$ & 23 & $127.1 \pm 15.2$ & $128.1 \pm 16.1$ \\
\hline Diastolic BP (mm Hg) & 30 & $79.7 \pm 11.0$ & $77.1 \pm 13.2$ & 22 & $80.6 \pm 11.2$ & $75.7 \pm 9.9$ \\
\hline Plasma glucose (mg/dL) & 29 & $188.8 \pm 67.1$ & $167.8 \pm 64.1$ & 23 & $184.0 \pm 54.0$ & $162.7 \pm 91.0$ \\
\hline HbA1c (\%) & 17 & $8.1 \pm 2.0$ & $7.4 \pm 1.2 * *$ & 12 & $8.5 \pm 1.0$ & $7.7 \pm 1.2 *$ \\
\hline AST (U/L) & 31 & $34.0 \pm 28.3$ & $30.8 \pm 22.0$ & 24 & $36.9 \pm 31.8$ & $24.0 \pm 13.6^{* *}$ \\
\hline $\operatorname{ALT}(\mathrm{U} / \mathrm{L})$ & 33 & $37.9 \pm 27.3$ & $37.2 \pm 43.6$ & 26 & $41.2 \pm 29.3$ & $29.6 \pm 23.8^{* *}$ \\
\hline$\gamma$-GT (U/L) & 26 & $50.3 \pm 33.0$ & $48.1 \pm 33.5$ & 21 & $51.0 \pm 30.6$ & $43.2 \pm 30.6$ \\
\hline Uric acid (mg/dL) & 20 & $5.8 \pm 1.5$ & $5.9 \pm 1.6$ & 16 & $5.6 \pm 1.3$ & $6.1 \pm 1.6$ \\
\hline TG (mg/dL) & 25 & $224.2 \pm 173.3$ & $261.5 \pm 284.5$ & 19 & $268.1 \pm 188.8$ & $276.2 \pm 269.8$ \\
\hline HDL-C (mg/dL) & 23 & $48.0 \pm 12.4$ & $49.3 \pm 10.8$ & 18 & $43.9 \pm 10.2$ & $47.9 \pm 11.2 * *$ \\
\hline LDL-C (mg/dL) & 16 & $111.9 \pm 42.7$ & $99.9 \pm 28.2$ & 15 & $110.2 \pm 32.3$ & $108.9 \pm 30.5$ \\
\hline Non-HDL-C (mg/dL) & 16 & $145.3 \pm 45.5$ & $141.5 \pm 46.1$ & 15 & $144.3 \pm 36.7$ & $151.9 \pm 38.0$ \\
\hline
\end{tabular}

${ }^{*} \mathrm{P}<0.1$ and ${ }^{* *} \mathrm{P}<0.05$ vs. baseline. 
Table 6. Changes in Metabolic Parameters at 3 and 6 Months After the Start of Luseogliflozin

\begin{tabular}{|c|c|c|c|c|c|c|}
\hline & $\mathbf{n}$ & $\begin{array}{l}\text { Baseline, } \\
\text { mean } \pm \text { SD }\end{array}$ & $\begin{array}{l}3 \text { months after, } \\
\text { mean } \pm \text { SD }\end{array}$ & $\mathbf{n}$ & $\begin{array}{l}\text { Baseline, } \\
\text { mean } \pm \text { SD }\end{array}$ & $\begin{array}{l}6 \text { months after, } \\
\text { mean } \pm \text { SD }\end{array}$ \\
\hline Body weight (kg) & 29 & $81.3 \pm 16.1$ & $79.8 \pm 16.1^{* *}$ & 21 & $81.5 \pm 15.6$ & $79.8 \pm 15.5^{* *}$ \\
\hline Systolic BP (mm Hg) & 27 & $137.4 \pm 20.8$ & $129.9 \pm 16.1^{*}$ & 21 & $134.7 \pm 23.3$ & $127.9 \pm 23.2$ \\
\hline Diastolic BP (mm Hg) & 27 & $83.9 \pm 13.4$ & $78.5 \pm 11.1^{* *}$ & 21 & $83.5 \pm 13.9$ & $77.2 \pm 10.2 * *$ \\
\hline Plasma glucose (mg/dL) & 32 & $201.8 \pm 95.0$ & $173.1 \pm 70.5^{*}$ & 21 & $202.7 \pm 94.0$ & $177.5 \pm 87.1^{*}$ \\
\hline $\mathrm{HbAlc}(\%)$ & 28 & $8.8 \pm 1.6$ & $8.0 \pm 1.3^{* *}$ & 20 & $8.7 \pm 1.8$ & $7.5 \pm 1.0 * *$ \\
\hline AST (U/L) & 34 & $36.6 \pm 29.4$ & $30.1 \pm 21.0^{* *}$ & 22 & $42.7 \pm 32.6$ & $27.8 \pm 19.6^{* *}$ \\
\hline ALT (U/L) & 35 & $47.0 \pm 41.9$ & $40.3 \pm 36.2 * *$ & 22 & $59.8 \pm 47.3$ & $43.9 \pm 39.6^{* *}$ \\
\hline$\gamma-\mathrm{GT}(\mathrm{U} / \mathrm{L})$ & 27 & $64.6 \pm 50.4$ & $50.3 \pm 41.1^{* *}$ & 20 & $73.8 \pm 49.1$ & $50.4 \pm 33.4 * *$ \\
\hline Uric acid (mg/dL) & 26 & $5.7 \pm 1.6$ & $5.2 \pm 1.6^{* *}$ & 15 & $6.0 \pm 1.2$ & $5.4 \pm 1.0^{* *}$ \\
\hline TG (mg/dL) & 30 & $213.5 \pm 163.2$ & $187.0 \pm 134.6^{*}$ & 19 & $284.7 \pm 174.5$ & $223.3 \pm 138.0^{* *}$ \\
\hline HDL-C (mg/dL) & 29 & $50.2 \pm 13.6$ & $51.2 \pm 13.3$ & 18 & $45.1 \pm 7.9$ & $45.4 \pm 6.5$ \\
\hline LDL-C (mg/dL) & 30 & $113.3 \pm 31.2$ & $110.4 \pm 38.4$ & 19 & $113.0 \pm 34.0$ & $106.0 \pm 36.7$ \\
\hline Non-HDL-C (mg/dL) & 24 & $146.2 \pm 47.6$ & $143.5 \pm 48.2$ & 15 & $163.5 \pm 41.9$ & $141.9 \pm 37.1^{* *}$ \\
\hline
\end{tabular}

${ }^{*} \mathrm{P}<0.1$ and ${ }^{* *} \mathrm{P}<0.05$ vs. baseline.

A 12-week dapagliflozin (10 mg/day) treatment exerted no significant effect on HDL-C levels [10]; however, a 6-month dapagliflozin treatment significantly elevated HDL-C levels in present study. In pooled analysis of phase 3 study results, relative to placebo, favorable changes in HDL-C and TG were seen with canagliflozin (26-week treatment); increases in LDL-C were also seen [11]. However, in our study, canagliflozin tended to decrease TG; however, any changes in both HDL-C and LDL-C were not observed.

A 12-week dapagliflozin treatment reduced serum uric acid in the study by List et al [12], which was not observed in our study. An 8-week tofogliflozin treatment $(\mathrm{n}=10)$ tended to decrease postprandial TG and significantly decreased uric acid, and a 16-week tofogliflozin $(\mathrm{n}=10)$ treatment significantly elevated HDL-C [13]. However, tofogliflozin did not affect liver function. In our study, tofogliflozin ameliorated liver function and uric acid, but did not show any influences on serum lipids. A 12-week ipragliflozin treatment $(n=257)$ significantly reduced body weight, systolic and diastolic BP, plasma glucose, HbA1c, TG, AST, ALT, $\gamma$-GT, uric acid, and elevated HDL-C, in the study by Iizuka et al [14]. However, ipragliflozin treatment $(n=23)$ reduced only body weight, HbA1c and AST. We obtained the similar results to those in Iizuka's study, by luseogliflozin treatment $(n=71)$, suggest-

Table 7. Changes in Metabolic Parameters at 3 and 6 Months After the Start of SGLT2 Inhibitors

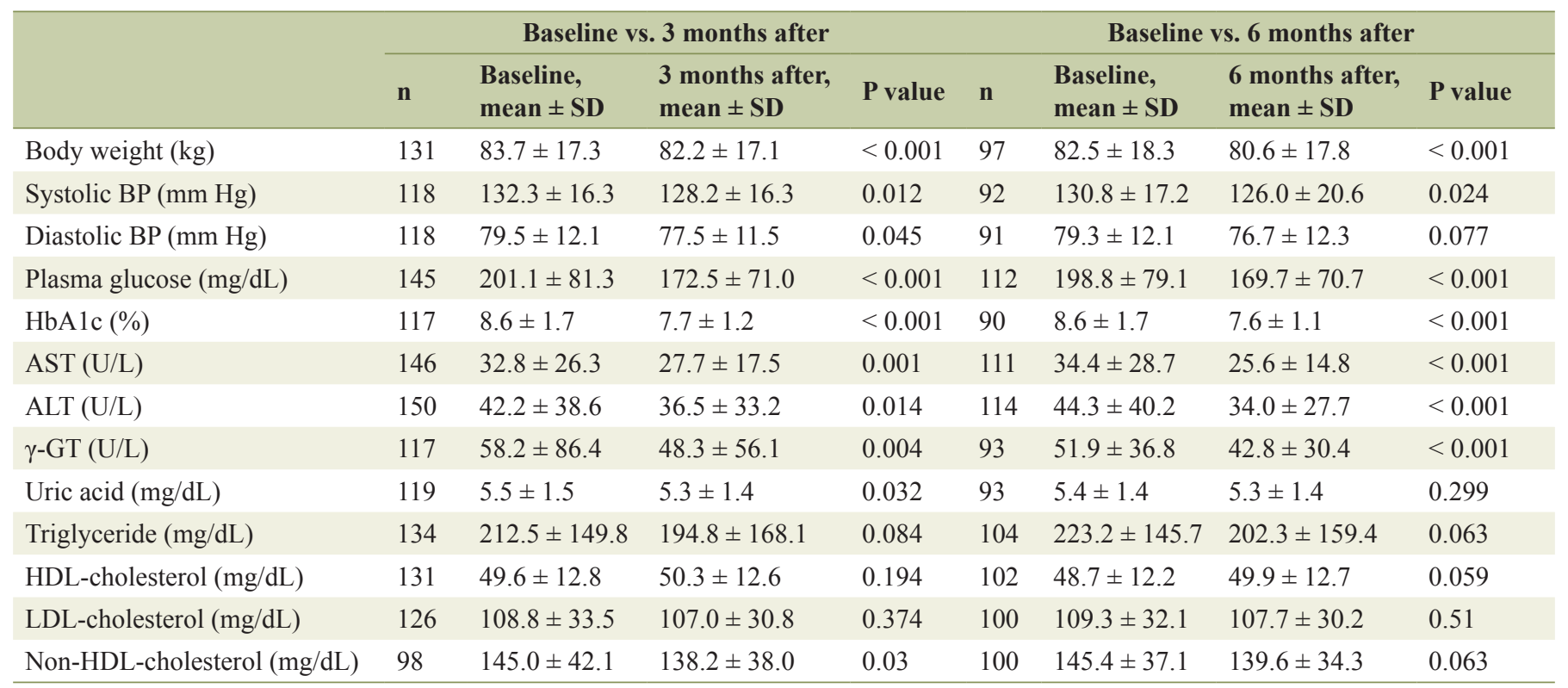


Table 8. Summary of Changes in Metabolic Parameters at 3 and 6 Months After the Start of Each SGLT2 Inhibitor

\begin{tabular}{llllll}
\hline SGLT2i Tofogliflozin & Canagliflozin & Empagliflozin & Ipragliflozin & Dapagliflozin Luseogliflozin
\end{tabular}

Body weight

$\begin{array}{llllllll}3 \text { months } & \downarrow & \downarrow & (\downarrow) & & \downarrow & \downarrow & \\ 6 \text { months } & \downarrow & \downarrow & (\downarrow) & \downarrow & (\downarrow) & \downarrow & \downarrow\end{array}$

Systolic BP

$\begin{array}{ccc}3 \text { months } & \downarrow & (\downarrow) \\ 6 \text { months } & \downarrow & \downarrow\end{array}$

Diastolic BP

$\begin{array}{lc}3 \text { months } & \downarrow \\ 6 \text { months } & (\downarrow)\end{array}$

Plasma glucose

$\begin{array}{llll}3 \text { months } & \downarrow & \downarrow & (\downarrow) \\ 6 \text { months } & \downarrow & \downarrow & \downarrow\end{array}$

$(\downarrow) \quad(\downarrow)$

6 months $\quad \downarrow \quad \downarrow \quad \downarrow \quad \downarrow \quad \downarrow$ ( $\quad \downarrow)$

$\mathrm{HbA} 1 \mathrm{c}$

$\begin{array}{llllllll}3 \text { months } & \downarrow & \downarrow & \downarrow & \downarrow & \downarrow & \downarrow & \downarrow \\ 6 \text { months } & \downarrow & (\downarrow) & \downarrow & \downarrow & \downarrow & (\downarrow) & \downarrow\end{array}$

AST

3 months $\quad \downarrow \quad(\downarrow)$

6 months

ALT

$\begin{array}{ll}3 \text { months } & \downarrow \\ 6 \text { months } & \downarrow\end{array}$

$\gamma$-GT

3 months

6 months

Uric acid

$\begin{array}{ll}3 \text { months } & \downarrow \\ 6 \text { months } & (\downarrow)\end{array}$

TG
3 months
$(\downarrow)$
$(\downarrow)$
$\downarrow$
6 months
$(\downarrow)$

$(\downarrow)$

HDL-C

3 months

6 months

$(\uparrow)$

$\uparrow$

$\downarrow$

$\downarrow$

)


Table 9. The Most Crucial Determinant in Baseline Parameters for Changes in Metabolic Parameters by Using SGLT2 Inhibitors

\begin{tabular}{|c|c|c|}
\hline Most crucial determinant & $\mathbf{r}$ & P value \\
\hline \multicolumn{3}{|l|}{ Changes in body weight } \\
\hline \multicolumn{3}{|l|}{ Systolic BP } \\
\hline 3 months & -0.205 & 0.02 \\
\hline 6 months & -0.293 & 0.004 \\
\hline \multicolumn{3}{|l|}{ Changes in systolic BP } \\
\hline \multicolumn{3}{|l|}{ Systolic BP } \\
\hline 3 months & -0.42 & $<0.001$ \\
\hline 6 months & 0.34 & $<0.001$ \\
\hline \multicolumn{3}{|l|}{ Changes in diastolic BP } \\
\hline \multicolumn{3}{|l|}{ Diastolic BP } \\
\hline 3 months & -0.425 & $<0.001$ \\
\hline 6 months & -0.611 & $<0.001$ \\
\hline \multicolumn{3}{|l|}{ Changes in plasma glucose } \\
\hline \multicolumn{3}{|l|}{ Plasma glucose } \\
\hline 3 months & -0.676 & $<0.001$ \\
\hline 6 months & -0.674 & $<0.001$ \\
\hline \multicolumn{3}{|l|}{ Changes in $\mathrm{HbAlc}$} \\
\hline \multicolumn{3}{|l|}{$\mathrm{HbAlc}$} \\
\hline 3 months & -0.619 & $<0.001$ \\
\hline 6 months & -0.659 & $<0.001$ \\
\hline \multicolumn{3}{|l|}{ Changes in AST } \\
\hline \multicolumn{3}{|l|}{ AST } \\
\hline 3 months & -0.582 & $<0.001$ \\
\hline 6 months & -0.023 & 0.023 \\
\hline \multicolumn{3}{|l|}{ Changes in ALT } \\
\hline \multicolumn{3}{|l|}{ ALT } \\
\hline 3 months & -0.441 & $<0.001$ \\
\hline 6 months & -0.429 & $<0.001$ \\
\hline \multicolumn{3}{|l|}{ Changes in $\gamma$-GT } \\
\hline \multicolumn{3}{|l|}{$\gamma-\mathrm{GT}$} \\
\hline 3 months & -0.517 & $<0.001$ \\
\hline \multicolumn{3}{|l|}{ ALT } \\
\hline 6 months & -0.478 & $<0.001$ \\
\hline \multicolumn{3}{|l|}{ Changes in uric acid } \\
\hline \multicolumn{3}{|l|}{ Uric acid } \\
\hline 3 months & -0.694 & $<0.001$ \\
\hline 6 months & -0.463 & $<0.001$ \\
\hline Changes in TG & & \\
\hline Non-HDL-C & & \\
\hline 3 months & -0.389 & $<0.001$ \\
\hline TG & & \\
\hline 6 months & -0.425 & $<0.001$ \\
\hline Changes in HDL-C & & \\
\hline LDL-C & & \\
\hline 3 months & -0.445 & $<0.001$ \\
\hline HDL-C & & \\
\hline 6 months & -0.428 & 0.019 \\
\hline Changes in LDL-C & & \\
\hline LDL-C & & \\
\hline 3 months & -0.351 & $<0.001$ \\
\hline 6 months & -0.353 & $<0.001$ \\
\hline Changes in non-HDL-C & & \\
\hline Non-HDL-C & & \\
\hline 3 months & -0.389 & $<0.001$ \\
\hline LDL-C & & \\
\hline 6 months & -0.458 & $<0.001$ \\
\hline
\end{tabular}

ing that the number of subjects is largely associated with the results by SGLT2i treatment.

In our previous study, we examined effects of SGLT2i on metabolic parameters in 50 patients with type 2 diabetes, and we found that SGLT2 $\mathrm{i}$ improved the glycemic control and reduced body weight and serum AST and ALT levels, whereas no significant changes were observed in serum lipids and uric acid [15]. However, in present study $(n=249)$, SGLT2is reduced serum TG, non-HDL-C and uric acid, and also increased serum HDL-C, supporting a significant influence of the number of subjects on results due to SGLT2i. We also think that the levels of metabolic parameters at baseline may affect changes in metabolic parameters. Briefly, the decrease in systolic BP due to SGLT2i is larger in patients with higher systolic BP at baseline. In our study, change in each metabolic parameter except for body weight was significantly correlated with each metabolic parameter at baseline, supporting our hypothesis. Controversial results in metabolic parameters may be due to difference in the number of participants, data at baseline and duration of treatment.

In terms of the occurrence of CV events due to SGLT2i, Monami et al performed a meta-analysis of RCTs, and found that treatment with SGLT2is was associated with a significant reduction in all-cause mortality, CV mortality, and myocardial infarction, but not stroke, with no apparent difference across molecules [5]. However, another meta-analysis of RCTs suggested that SGLT2 $\mathrm{i}$ appeared to reduce both all-cause and CV mortality, and the benefit was only seen with empagliflozin, and potential harm was observed with dapagliflozin [6], challenging a class effect of SGLT2i [5]. However, the meta-analysis by Tang et al denied any harm associated with dapagliflozin [7]. A recent systematic review and meta-analysis by Wu et al reported that SGLT2i protected against the risk of major adverse CV events, CV death, heart failure, and death from any cause, and no clear effect on non-fatal myocardial infarction or angina, and an adverse effect for non-fatal stroke [8].

McGovern et al performed a cross-sectional analysis of $\mathrm{CV}$ risk in patients with type 2 diabetes and a subgroup prescribed SGLT2i to identify and describe the proportion of patients with type 2 diabetes who have the comparable high $\mathrm{CV}$ risk to those included in the EMPA-REG trial, and they concluded that the EMPA-REG trial results are applicable only to a small proportion of patients with type 2 diabetes and additional data are required [16], suggesting also an importance of backgrounds of subjects at baseline.

The present study has several limitations. First, other hypoglycemic, anti-hypertensive, or lipid lowering agents, food intakes and/or exercise levels may have an influence on the study results. Second, the number of studied subjects was small because of the limited availability. Third, since the study was based on charts, lack of data might influence the results. A more detailed prospective study is recommended to evaluate the effects of SGLT2i on metabolic parameters more validly.

The present study also has the strength. First, metabolic parameters were measured by the same laboratory. Second, subjects were treated by the specialists for diabetes treatment who were equally educated by the same specialist. Third, to our knowledge, this study is the first to show effects of six SGLT2is on metabolic parameters in patients with type 2 dia- 
betes.

\section{Conclusion}

Our study demonstrated that SGLT2i ameliorated body weight, systolic and diastolic BP, liver function, serum lipids and uric acid, in addition to improvement of glucose metabolism.

\section{Author Contributions}

$\mathrm{HY}, \mathrm{MH}$, and HK designed the research. HA, AK, YW, TH, YM, KK, HH, and AS collected data. HY and TK analyzed data, and HY wrote the paper. All authors read and approved the final paper.

\section{Competing Interests}

The authors declare that they have no competing interest concerning this article.

\section{References}

1. Vallon V, Platt KA, Cunard R, Schroth J, Whaley J, Thomson SC, Koepsell H, et al. SGLT2 mediates glucose reabsorption in the early proximal tubule. J Am Soc Nephrol. 2011;22(1):104-112.

2. Jabbour SA, Goldstein BJ. Sodium glucose co-transporter 2 inhibitors: blocking renal tubular reabsorption of glucose to improve glycaemic control in patients with diabetes. Int J Clin Pract. 2008;62(8):1279-1284.

3. Yanai H, Katsuyama H, Hamasaki H, Adachi H, Moriyama S, Yoshikawa R, Sako A. Sodium-Glucose Cotransporter 2 Inhibitors: Possible Anti-Atherosclerotic Effects Beyond Glucose Lowering. J Clin Med Res. 2016;8(1):1014.

4. Zinman B, Wanner C, Lachin JM, Fitchett D, Bluhmki E, Hantel S, Mattheus M, et al. Empagliflozin, Cardiovascular Outcomes, and Mortality in Type 2 Diabetes. N Engl J Med. 2015;373(22):2117-2128.

5. Monami M, Dicembrini I, Mannucci E. Effects of SGLT2 inhibitors on mortality and cardiovascular events: a comprehensive meta-analysis of randomized controlled trials. Acta Diabetol. 2017;54(1):19-36.

6. Saad M, Mahmoud AN, Elgendy IY, Abuzaid A, Barakat AF, Elgendy AY, Al-Ani M, et al. Cardiovascular outcomes with sodium-glucose cotransporter-2 inhibitors in patients with type II diabetes mellitus: A meta-analysis of placebo-controlled randomized trials. Int J Cardiol. 2017;228:352-358.

7. Tang H, Fang Z, Wang T, Cui W, Zhai S, Song Y. MetaAnalysis of Effects of Sodium-Glucose Cotransporter 2 Inhibitors on Cardiovascular Outcomes and All-Cause Mortality Among Patients With Type 2 Diabetes Mellitus. Am J Cardiol. 2016;118(11):1774-1780.

8. Wu JH, Foote C, Blomster J, Toyama T, Perkovic V, Sundstrom J, Neal B. Effects of sodium-glucose cotransporter-2 inhibitors on cardiovascular events, death, and major safety outcomes in adults with type 2 diabetes: a systematic review and meta-analysis. Lancet Diabetes Endocrinol. 2016;4(5):411-419.

9. Matsuo S, Imai E, Horio M, Yasuda Y, Tomita K, Nitta $\mathrm{K}$, Yamagata $\mathrm{K}$, et al. Revised equations for estimated GFR from serum creatinine in Japan. Am J Kidney Dis. 2009;53(6):982-992.

10. Fadini GP, Bonora BM, Zatti G, Vitturi N, Iori E, Marescotti MC, Albiero M, et al. Effects of the SGLT2 inhibitor dapagliflozin on HDL cholesterol, particle size, and cholesterol efflux capacity in patients with type 2 diabetes: a randomized placebo-controlled trial. Cardiovasc Diabetol. 2017;16(1):42.

11. Usiskin K, Kline I, Fung A, Mayer C, Meininger G. Safety and tolerability of canagliflozin in patients with type 2 diabetes mellitus: pooled analysis of phase 3 study results. Postgrad Med. 2014;126(3):16-34.

12. List JF, Woo V, Morales E, Tang W, Fiedorek FT. Sodium-glucose cotransport inhibition with dapagliflozin in type 2 diabetes. Diabetes Care. 2009;32(4):650-657.

13. Kakuda H, Kobayashi J, Sakurai M, Kakuda M, Takekoshi N. The Effect of Tofogliflozin Treatment on Postprandial Glucose and Lipid Metabolism in Japanese Men With Type 2 Diabetes: A Pilot Study. J Clin Med Res. 2017;9(5):403-409.

14. Iizuka T, Iemitsu $\mathrm{K}$, Takihata M, Takai M, Nakajima S, Minami N, Umezawa S, et al. Efficacy and Safety of Ipragliflozin in Japanese Patients With Type 2 Diabetes: Interim Outcome of the ASSIGN-K Study. J Clin Med Res. 2016;8(2):116-125.

15. Katsuyama H, Hamasaki H, Adachi H, Moriyama S, Kawaguchi A, Sako A, Mishima S, et al. Effects of Sodium-Glucose Cotransporter 2 Inhibitors on Metabolic Parameters in Patients With Type 2 Diabetes: A Chart-Based Analysis. J Clin Med Res. 2016;8(3):237-243.

16. McGovern A, Feher M, Munro N, de Lusignan S. Sodium-Glucose Co-transporter 2 (SGLT2) Inhibitor: Comparing Trial Data and Real-World Use. Diabetes Ther. 2017;8(2):365-376. 